\title{
(C) OPEN ACCESS \\ Risk-adjusted survival for adults following in-hospital cardiac arrest by day of week and time of day: observational cohort study
}

\author{
Emily J Robinson, ' Gary B Smith, ${ }^{2}$ Geraldine S Power, ${ }^{1}$ David A Harrison, ${ }^{1}$ \\ Jerry Nolan, ${ }^{3}$ Jasmeet Soar, ${ }^{4}$ Ken Spearpoint, ${ }^{5}$ Carl Gwinnutt, ${ }^{6}$ \\ Kathryn M Rowan ${ }^{1}$
}

- Additional material is published online only. To view please visit the journal online (http://dx.doi.org/10.1136/bmjgs2015-004223).

For numbered affiliations see end of article.

\section{Correspondence to} Professor Gary B Smith, Faculty of Health and Social Sciences, University of Bournemouth,

Royal London House, Christchurch Road, Bournemouth BH1 3LT, UK; gbsresearch@ virginmedia.com

Received 21 March 2015 Revised 27 October 2015 Accepted 9 November 2015 Published Online First 11 December 2015

\section{SLinked}

- http://dx.doi.org/10.1136/ bmjqs-2015-005037

\section{CrossMark}

To cite: Robinson EJ, Smith GB, Power GS, et al. BMJ Qual Saf 2016;25:832841.

\begin{abstract}
Background Internationally, hospital survival is lower for patients admitted at weekends and at night. Data from the UK National Cardiac Arrest Audit (NCAA) indicate that crude hospital survival was worse after in-hospital cardiac arrest (IHCA) at night versus day, and at weekends versus weekdays, despite similar frequency of events.

Objective To describe IHCA demographics during three day/time periods - weekday daytime (Monday to Friday, 08:00 to 19:59), weekend daytime (Saturday and Sunday, 08:00 to 19:59) and night-time (Monday to Sunday, 20:00 to 07:59) —and to compare the associated rates of return of spontaneous circulation (ROSC) for $>20 \mathrm{~min}$ (ROSC>20 min) and survival to hospital discharge, adjusted for risk using previously developed NCAA risk models. To consider whether any observed difference could be attributed to differences in the case mix of patients resident in hospital and/or the administered care.
\end{abstract}

Methods We performed a prospectively defined analysis of NCAA data from 27700 patients aged $\geq 16$ years receiving chest compressions and/or defibrillation and attended by a hospitalbased resuscitation team in response to a resuscitation (2222) call in 146 UK acute hospitals

Results Risk-adjusted outcomes (OR (95\% CI)) were worse $(p<0.001)$ for both weekend daytime (ROSC > 20 min 0.88 (0.81 to 0.95 ); hospital survival $0.72(0.64$ to 0.80$))$, and nighttime (ROSC>20 min 0.72 (0.68 to 0.76); hospital survival 0.58 (0.54 to 0.63$)$ ) compared with weekday daytime. The effects were stronger for non-shockable than shockable rhythms, but there was no significant interaction between day/ time of arrest and age, or day/time of arrest and arrest location. While many daytime IHCAs involved procedures, restricting the analyses to IHCAs in medical admissions with an arrest location of ward produced results that are broadly in line with the primary analyses.

Conclusions IHCAs attended by the hospitalbased resuscitation team during nights and weekends have substantially worse outcomes than during weekday daytimes. Organisational or care differences at night and weekends, rather than patient case mix, appear to be responsible.

\section{BACKGROUND}

Internationally, hospital survival is lower for patients admitted at weekends and at night. ${ }^{1-8}$ The effect exists for a range of diagnoses, including several cardiological conditions. ${ }^{1} 2478$ Concha et al ${ }^{4}$ found that for patients admitted at weekends with cardiac arrest and arrhythmia the majority of excess deaths occurred within the first $24 \mathrm{~h}$ after admission, suggesting that the different survival between weekday and weekend admissions is most likely due to variations in care.

While many studies consider night/ weekend effects in relation to day of hospital admission, there is less data for events occurring in hospital. ${ }^{9}{ }^{10}$ A few, generally small, single-centre studies from outside the UK have reported reduced survival for in-hospital cardiac arrests (IHCAs) occurring outside weekday hours. ${ }^{11-16}$ Recent analysis of UK National Cardiac Arrest Audit (NCAA) data demonstrated reduced crude hospital survival after IHCA at night versus day, and at weekends versus weekdays, despite a similar frequency of events. ${ }^{17}$ In the USA, risk-adjusted outcomes from the 
American Heart Association's (AHA) 'Get With The Guidelines-Resuscitation' (GWTG-R) registry of IHCA indicate that hospital survival was substantially lower at nights and weekends compared with weekday daytimes. ${ }^{18}$ However, extrapolating results from the US to UK practice may not be justified, as a high proportion of patients (up to 85\%) in the GWTG-R registry had a monitored IHCA (up to 59\% in an intensive care unit (ICU)) $)^{18} 19$ and the UK has fewer ICU beds. ${ }^{20}$

As it is not known if risk-adjusted outcomes for IHCA occurring at weekends and at night in UK acute hospitals are worse than for those occurring during weekday daytime, we investigated this using the NCAA database and the NCAA risk models for IHCA outcomes. $^{21}$ We also attempted to discover if any observed difference was attributable to the case mix of patients resident in hospital or to care delivery.

\section{METHODS}

\section{UK National Cardiac Arrest Audit}

NCAA is the UK national clinical audit for patients, aged $>28$ days, who receive cardiopulmonary resuscitation (CPR) following an IHCA attended by a hospital-based resuscitation team (or equivalent) in response to a 2222 call (2222 is the emergency telephone number used to summon a resuscitation team in UK National Health Service (NHS) hospitals). NCAA defines CPR as the receipt of chest compressions and/or defibrillation. NCAA has approval from the Confidentiality Advisory Group within the Health Research Authority to hold patient identifiable data under Section 251 of the NHS Act 2006 (approval number: ECC 2-06(n)/2009). At the time of the study, 163 hospitals in England, Wales, Scotland and Northern Ireland participated in NCAA, with coverage in England representing $>78 \%$ of adult, acute hospitals.

\section{NCAA database}

Standardised data are collected, both at the time of the IHCA and from medical records, according to strict rules and definitions (these have been reported previously ${ }^{17}$ ). NCAA data are entered onto a dedicated, secure, online data entry system by relevant staff at participating hospitals. Data are validated both locally (at the point of data entry) and centrally for completeness, illogicalities and inconsistencies. For consistency, day and time of 2222 call are, hereafter, termed day and time of IHCA.

\section{Inclusion and exclusion criteria}

Validated data for the period 1 April 2011 to 30 September 2013 were selected for analysis. All IHCAs for adult patients (aged $\geq 16$ years) were included. The following were excluded: IHCA data from specialist, non-acute or paediatric hospitals; prehospital cardiac arrests (included in the NCAA if the resuscitation team is called to the emergency department to continue the resuscitation attempt); patients missing important data for date/time of IHCA, age, risk factors and outcome; and any subsequent IHCA in a given hospital stay for the same patient. We also excluded IHCAs where the patient had a Do Not Attempt Cardio-Pulmonary Resuscitation (DNACPR) decision in place before the arrest as this is an explicit exclusion from the NCAA risk models used to 'risk adjust' the outcomes studied (see below).

\section{Data}

The following data were extracted for all included IHCAs: age, sex, prior hospital length of stay, reason for admission to/attendance at hospital, location of IHCA, presenting/first documented rhythm, return of spontaneous circulation (ROSC) for $>20 \mathrm{~min}$ (ROSC $>20 \mathrm{~min}$ ), survival to hospital discharge and reason resuscitation was stopped.

Prior hospital length of stay was defined as the number of days between hospital admission and the date of the IHCA and was categorised as $0,1,2-7$ and $\geq 8$ days. Presenting/first documented rhythm was characterised as one of ventricular fibrillation (VF), pulseless ventricular tachycardia (pVT), shockable unknown rhythm, asystole, pulseless electrical activity (PEA), bradycardia, non-shockable unknown rhythm or unknown or undetermined rhythm. ROSC $>20 \mathrm{~min}$ and survival to hospital discharge were the primary outcomes. Locations were grouped as follows:

- ward, obstetric unit or intermediate care setting

- emergency department

- emergency admissions unit

- operating theatre and theatre recovery

- cardiac catheterisation laboratory

- imaging department or specialist treatment area

- critical care unit (ie, intensive care or high dependency unit)

- clinic or non-clinical area.

\section{Categorisation of day and time of IHCA}

Day and time of IHCA were categorised as:

1. weekday daytime (Monday to Friday, 08:00 to 19:59)

2. weekend daytime (Saturday and Sunday, 08:00 to 19:59)

3. night-time (Monday to Sunday, 20:00 to 07:59).

These periods were chosen a priori, based on definitions used in a previous NCAA publication. ${ }^{8}$

\section{Statistical analyses}

Descriptive statistics

We made an a priori decision to calculate IHCA rate per 1000 hospital admissions (pooled across all hospitals) per $12 \mathrm{~h}$, based upon definitions from a previous NCAA publication. ${ }^{17}$ Case mix and outcomes were described for each of the three day/time periods: weekday daytime, weekend daytime and night-time. Predicted probabilities for the two primary outcomes, 
ROSC $>20$ min and survival to hospital discharge, were calculated. Categorical data were summarised as number and percentage, continuous data as mean (SD) or median (IQR), as appropriate.

Logistic regression analysis

An unadjusted logistic regression model was run to estimate the association between the three day/time periods and each of the two primary outcomes. The association was reported as the OR for each outcome for weekend daytime and for night-time compared with weekday daytime as the baseline category. These associations were then reassessed after adjustment for the risk factors included in the NCAA risk prediction models, ${ }^{21}$ that is, age, sex (in the ROSC $>20 \mathrm{~min}$ model only), prior hospital length of stay, reason for admission to/attendance at hospital, location of IHCA and presenting/first documented rhythm. Information about the categorisation of the included variables is provided in an earlier NCAA publication ${ }^{21}$ and in online supplementary files 1 and 2 . The development set for the NCAA models contained 14688 patients with an IHCA, of which 6605 (45.0\%) achieved ROSC > $20 \mathrm{~min}$ and 2926 (19.9\%) survived to hospital discharge. ${ }^{21}$ Predicted probabilities for ROSC $>20 \mathrm{~min}$ and survival to hospital discharge were calculated for each arrest. A hospital-level random effect was included in the models to adjust for clustering of outcomes at the hospital level.

Wald tests were used to test the global and pairwise comparisons between the day/time periods for any difference in crude and risk-adjusted outcomes. To assess whether the association between day/time periods and outcomes varied across specific subgroups, interactions between day/time period and the following covariates were tested: age group $(16-64,65-74,75-84$, $85+$ ); presenting/first documented rhythm (shockable rhythm, asystole, PEA, other non-shockable or unknown rhythm) and location of IHCA. The prospective decision to investigate these covariates was based on the view of the NCAA Steering Group that these could be clinically important.

A statistical analysis plan was agreed a priori. The analyses were performed using Stata/SE V.13.0 (StataCorp LP, Texas, USA).

\section{RESULTS}

During the period 1 April 2011 to 30 September 2013, a total of 36292 IHCAs from 159 hospitals were recorded in the NCAA database. After applying the study's inclusion and exclusion criteria (figure 1), 27700 IHCAs in 146 acute hospitals were included in the analysis. Only 105 IHCAs $(0.38 \%$ of the eligible data set) were excluded because of missing data.

The distribution of day/time of IHCA throughout the week is shown in figure 2. IHCAs were distributed equally between night-time $(13758,49.7 \%)$ and daytime (13 942，50.3\%), with $36.5 \%$ occurring during weekday daytime and $13.8 \%$ during weekend daytime (table 1). The mean rate of IHCA per 1000 hospital admissions per $12 \mathrm{~h}$ was similar for the selected day/time periods. Demographics and case mix, by day/time of IHCA, are presented in table 1 . Mean age, the proportion of males and the reason for admission to/attendance at hospital for patients having an IHCA were similar for day/time period. Across all day/time periods, approximately $80 \%$ of IHCAs occurred in medical patients and $>50 \%$ of IHCAs occurred on general hospital wards (table 1). Presenting rhythm was shockable (VF/pVT) in $15.4 \%$ and non-shockable (asystole, PEA, bradycardia) in $74.9 \%$ of arrests (table 1). Shockable rhythms were less common at night-time than during the daytime, whereas non-shockable rhythms were more common at night-time (table 1).

Distributions for the predicted probability of ROSC $>20$ min and for hospital survival, estimated from the NCAA risk prediction models, were similar across the day/time periods (table 1). However, crude survival for both outcomes was significantly lower $(\mathrm{p}<0.001)$ at night-time and weekend daytime compared with weekday daytime (figure 3A). After risk adjustment, both ROSC $>20 \mathrm{~min}$ and hospital survival remained significantly worse $(p<0.001)$ for both weekend daytime and for night-time compared with weekday daytime (figure 3B).

To ensure that our findings were not biased by (a) the influence of patients undergoing procedures (who generally have a greater chance of surviving an IHCA) being more likely to be present in hospital during the weekday daytime or (b) our daytime definition of 08:00 to 19:59, post hoc we performed additional separate analyses (i) restricted to medical admissions with a location of arrest on a ward and (ii) using a different daytime definition of 08:00-16:29. Restricting the data set produced results that are broadly in line with the primary analyses, except that the effect of weekend daytime versus weekday daytime disappeared for ROSC $>20 \mathrm{~min}$ (but remained for hospital survival) (see online supplementary file 3 ).

There was no significant interaction between day/ time of IHCA and patient age $(p=0.07$ for ROSC $>20 \mathrm{~min}, \mathrm{p}=0.21$ for hospital survival) or between day/time and location of IHCA ( $p=0.08$ for ROSC $>20$ min, $p=0.23$ for hospital survival). The effect of night and weekend was stronger for nonshockable than for shockable rhythms $(\mathrm{p}<0.001)$ (figure 4).

\section{DISCUSSION}

\section{Primary findings}

In NHS hospitals participating in the NCAA, rates of ROSC $>20$ min and survival to hospital discharge following IHCA were significantly worse for both nighttime and weekend daytime compared with weekday daytime, even after risk adjustment. Additional 


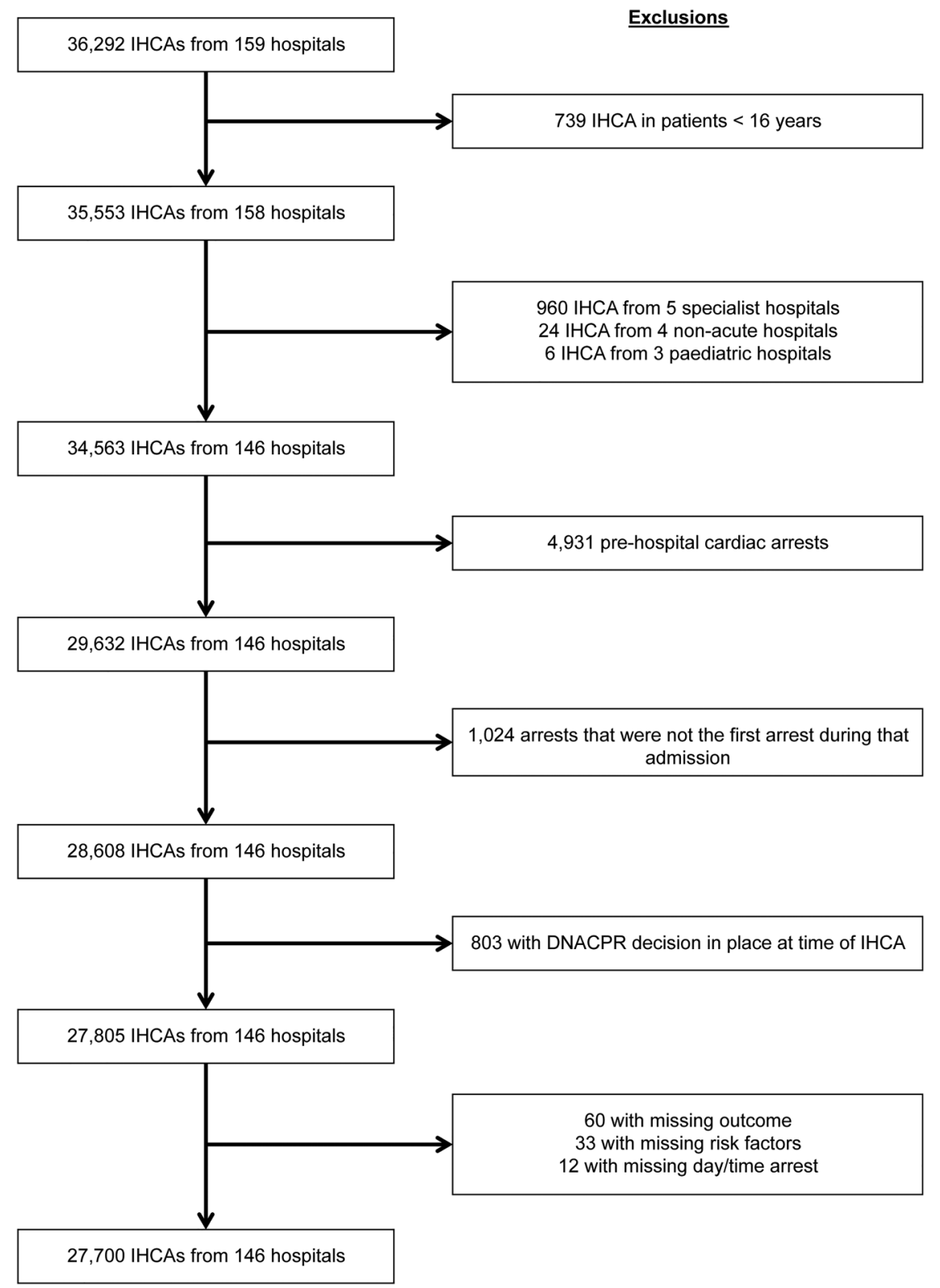

Figure 1 STROBE diagram for the study. IHCA, in-hospital cardiac arrest.

sensitivity analyses restricted to medical admissions and using an alternative definition for daytime both show that the magnitude of the weekend/night-time effect remains essentially unchanged. Importantly, procedure-related arrests do not have a major effect on the better outcomes seen during weekday daytime IHCAs. Overall, patients who have an arrest at night and weekend daytime have virtually half the chance of surviving to hospital discharge than those arresting at weekday daytime.

\section{Strengths/weaknesses of the study}

Major strengths of our study include a large representative sample of UK hospitals; clinical data collected to a high standard according to strict rules and definitions, validated locally and centrally; and a low level of missing data $(<0.4 \%$ of the eligible data set). That (a) mean rates of IHCA and (b) predicted probabilities for the two study outcomes were similar across the selected day/time periods are also important strengths. The first because it suggests that the number of IHCAs followed by ROSC $>20 \mathrm{~min}$ and survival to hospital discharge should be similar across time periods, and that selection bias is less likely to affect the results. The second because it demonstrates that the case mix of patients having an IHCA across the three time periods was similar, and that residual confounding due to unmeasured patient factors is less likely to affect the results than if large differences existed in the observed patient characteristics.

A major weakness of our study is that neither the NCAA database nor the models include every case-mix factor that may be important for patient outcome following IHCA. Specifically, NCAA does not collect data 


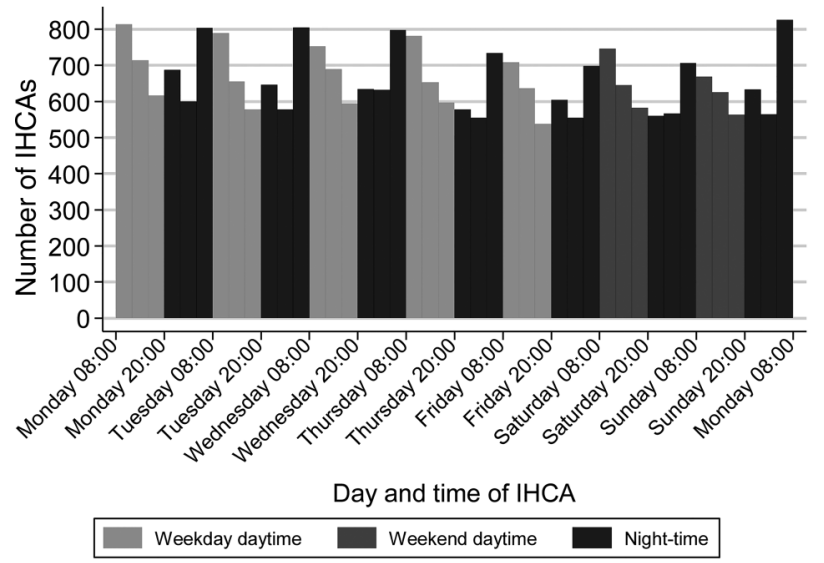

Figure 2 Distribution of day and time of in-hospital cardiac arrest (IHCA).

on patient comorbidities, which is a significant risk factor in the US model for post-IHCA outcomes. ${ }^{22}$ Equally, NCAA has no data on the diagnosis of patients having an IHCA, yet several researchers have demonstrated variation in excess mortality in patients admitted at weekends according to the patient's diagnostic group. ${ }^{12}{ }^{4}$ The inclusion of age and prior length of stay in hospital in the NCAA models might counterbalance these weaknesses as older patients have more comorbidity and patients with comorbidities experience longer hospital stays. ${ }^{23}{ }^{24}$ Both patient age and prior length of stay in hospital are similar between groups in our study (table 1). However, the lack of detailed case-mix data potentially reduces the significance of our findings. Another potential weakness is that the remit of the NCAA is to audit the outcomes of the resuscitation team (or equivalent) and, therefore, the database includes only IHCAs involving a 2222 call. Patients having an IHCA in a monitored area with specialist staff already present (eg, the ICU) may well be resuscitated without a 2222 call having been made or the hospital-based resuscitation team attending. Consequently, we are unable to compare outcomes where the patient received CPR following a 2222 call with those where no call was made.

\section{Other adjusted studies of IHCA outcomes by day/night}

Our results concur with those from other published adjusted analyses of outcomes by day and time of IHCA. ${ }^{11} 18$ In the large multicentre study published by the AHA GWTG-R registry, ${ }^{18}$ hospital survival following IHCA was lower during nights and weekends compared with day/evening on weekdays, even after adjustment for patient, arrest event and hospital factors. Similarly, data from a single Scandinavian centre also showed that, after adjustment for differences in age, history and factors at resuscitation, survival rate for patients having IHCAs during office hours was more than twice that of patients having an arrest during other times. ${ }^{11}$ The similarity between the NCAA and AHA findings is particularly interesting given that up to $85 \%$ of IHCAs in the AHA GWTG-R registry occurred in monitored areas of hospitals, up to $59 \%$ in an ICU. ${ }^{18}{ }^{19}$ Approximately $9.0 \%$ of acute care hospital beds in the USA are adult ICU beds compared with $1.2 \%$ in the UK. ${ }^{20}$

\section{Comparison with other publications: reported rhythms by day/night}

We found that non-shockable rhythms were more common at night-time than during the daytime (table 1); shockable rhythms had the opposite relationship. The weekend/night-time effect was also stronger for nonshockable than for shockable presenting rhythms (figure 4). In general, survival from shockable cardiac arrest rhythms is greater than for non-shockable rhythms ${ }^{17} 18$ and a shockable rhythm that is not treated within minutes may progress to a nonshockable rhythm. Therefore, the difference between the daytime and night-time incidence of shockable and non-shockable rhythms can have a biological causation or be due to an increased incidence of shockable rhythms progressing to nonshockable rhythms.

Many studies report that the presenting or first documented rhythm is more commonly shockable during the day, with those at night being more commonly non-shockable. ${ }^{11-14} 1718$ In our study, IHCAs during night-time were generally less likely to be associated with ROSC $>20$ min and survival to hospital discharge compared with weekday and weekend daytime. Swedish research reported better survival to hospital discharge for IHCA occurring from 08:00$16: 30$ compared with $16: 30-08: 00$, regardless of the initial arrest rhythm. ${ }^{11}$ Data from $>4500$ arrests at a single American hospital suggested that the observed survival difference between day and night was explained by circadian changes in initial rhythm. ${ }^{14}$ The authors reported that a significantly higher proportion of arrests had a presenting or first documented rhythm of asystole at night than during the day. In the UK, survival on general medical wards was worse for patients with a shockable rhythm occurring during late (15:30-21:00) and night shifts (21:00-17:30) compared with early shifts $(07: 30-15: 30){ }^{15}$

\section{Comparison with other publications: age and location}

We found no significant interaction with age and location of arrest. Our findings on age are similar to other authors. ${ }^{14} 18$ Our findings on location differ from those reported by the AHA where survival from IHCA at night was lower for all locations except the emergency department. ${ }^{18}$ Location of IHCA can act as a proxy for the likelihood of a patient being monitored at the time of the IHCA or the likelihood of the IHCA being witnessed. In Sweden, on monitored wards, the crude rate of survival to hospital discharge 
Table 1 Demographics and case mix by day/time of IHCA

\begin{tabular}{|c|c|c|c|}
\hline & Weekday daytime & Weekend daytime & Night-time \\
\hline Number of IHCAs, n (\%) & $10113(36.5)$ & $3829(13.8)$ & $13758(49.7)$ \\
\hline Rate per 1000 hospital admissions per $12 \mathrm{~h}$, mean $(\mathrm{Cl})$ & $0.135(0.133$ to 0.138$)$ & $0.127(0.123$ to 0.131$)$ & $0.131(0.129$ to 0.133$)$ \\
\hline Age mean (SD) & $73.4(14.2)$ & $73.8(14.3)$ & $74.0(14.4)$ \\
\hline Sex males (\%) & $5724(56.6)$ & $2144(56.0)$ & $7943(57.7)$ \\
\hline \multicolumn{4}{|l|}{ Length of stay in hospital prior to IHCA, n (\%) (N=27 693) } \\
\hline 0 days & $3405(33.7)$ & $1181(30.9)$ & $2915(21.2)$ \\
\hline 1 day & $1531(15.2)$ & $553(14.4)$ & $2653(19.3)$ \\
\hline 2-7 days & $2919(28.9)$ & $1218(31.8)$ & $4813(35.0)$ \\
\hline$\geq 8$ days & $2253(22.3)$ & $876(22.9)$ & $3376(24.5)$ \\
\hline \multicolumn{4}{|c|}{ Reason for admission to/attendance at/visit to hospital, n (\%) (N=27 694) } \\
\hline Medical & $7993(79.1)$ & $3139(82.0)$ & $11373(82.7)$ \\
\hline Trauma & $372(3.7)$ & $158(4.1)$ & $529(3.8)$ \\
\hline Elective/scheduled surgery & $696(6.9)$ & $201(5.3)$ & $692(5.0)$ \\
\hline Emergency/urgent surgery & $737(7.3)$ & $300(7.8)$ & $1107(8.1)$ \\
\hline Obstetric & $18(0.2)$ & $5(0.1)$ & $33(0.2)$ \\
\hline Outpatient & $249(2.5)$ & $16(0.4)$ & $14(0.1)$ \\
\hline Staff/visitor & $45(0.4)$ & $9(0.2)$ & $8(0.1)$ \\
\hline \multicolumn{4}{|l|}{ Location of IHCA n (\%) (N=27 698) } \\
\hline Ward, obstetrics or intermediate care & $5355(53.0)$ & $2231(58.3)$ & $8746(63.6)$ \\
\hline Emergency department & $1145(11.3)$ & $473(12.4)$ & $1224(8.9)$ \\
\hline Emergency admissions unit & $850(8.4)$ & $306(8.0)$ & $1242(9.0)$ \\
\hline Theatre and recovery & $228(2.3)$ & $47(1.2)$ & $85(0.6)$ \\
\hline Cardiac catheterisation laboratory & $466(4.6)$ & $86(2.2)$ & $161(1.2)$ \\
\hline Imaging department or specialist treatment & $519(5.1)$ & $90(2.4)$ & $121(0.9)$ \\
\hline ICU, ICU/HDU or HDU & $463(4.6)$ & $218(5.7)$ & $891(6.5)$ \\
\hline Coronary care unit & $918(9.1)$ & $366(9.6)$ & $1265(9.2)$ \\
\hline Clinic or non-clinical area & $168(1.7)$ & $12(0.3)$ & $22(0.2)$ \\
\hline \multicolumn{4}{|l|}{ Presenting/first documented rhythm, $n(\%)(\mathrm{N}=27 \mathrm{700})$} \\
\hline Shockable-VF & $1322(13.1)$ & $420(11.0)$ & $1234(9.0)$ \\
\hline Shockable-pVT & $536(5.3)$ & $192(5.0)$ & $557(4.0)$ \\
\hline Shockable—unknown & $64(0.6)$ & $27(0.7)$ & $64(0.5)$ \\
\hline Non-shockable-asystole & $1883(18.6)$ & $779(20.3)$ & $3959(28.8)$ \\
\hline Non-shockable-PEA & $5264(52.1)$ & $2014(52.6)$ & $6729(48.9)$ \\
\hline Non-shockable—bradycardia & $63(0.6)$ & $16(0.4)$ & $36(0.3)$ \\
\hline Non-shockable—unknown & $214(2.1)$ & $83(2.2)$ & $285(2.1)$ \\
\hline Unknown & $767(7.6)$ & $298(7.8)$ & $894(6.5)$ \\
\hline \multicolumn{4}{|c|}{ Predicted probabilities (\%) from the NCAA risk models median (IQR) $(\mathrm{N}=27689)$} \\
\hline $\mathrm{ROSC}>20 \mathrm{~min}$ & 43 (34 to 62 ) & 41 (33 to 59) & $43(28$ to 55$)$ \\
\hline Hospital survival & 13 (7 to 30$)$ & 12 (6 to 25$)$ & 10 (5 to 21$)$ \\
\hline
\end{tabular}

HDU, high dependency unit; ICU, intensive care unit; IHCA, in-hospital cardiac arrest-day/time categorised by 2222 call; NCAA, National Cardiac Arrest Audit; PEA, pulseless electrical activity; pVT, pulseless ventricular tachycardia; ROSC $>20 \mathrm{~min}$, return of spontaneous circulation for $>20$ min; VF, ventricular fibrillation.

was 50\% during office hours and 32\% after office hours. Corresponding figures for unmonitored wards were $48 \%$ and $21 \%$, respectively. ${ }^{11}$ Others report that IHCAs at night are more likely to be unwitnessed $^{12} 14 \quad 16$ and that unwitnessed arrests are associated with lower survival to hospital discharge. ${ }^{16}$ However, one group found no difference in ROSC $>20$ min or survival to hospital discharge for witnessed versus unwitnessed cases, either within or between night, morning or evening shifts. ${ }^{12}$

\section{Possible causes of poorer IHCA outcomes at night and} weekend

Our observation that IHCAs during night-time and weekend daytime have worse outcomes resonates with numerous reports of lower survival following weekend or out-of-hours hospital admission, ${ }^{1-8}$ even though the focus of our study is different. We did not consider day of hospital admission; rather, we studied IHCA outcomes at weekday daytime, weekend daytime and night-time. Patients in our study might 


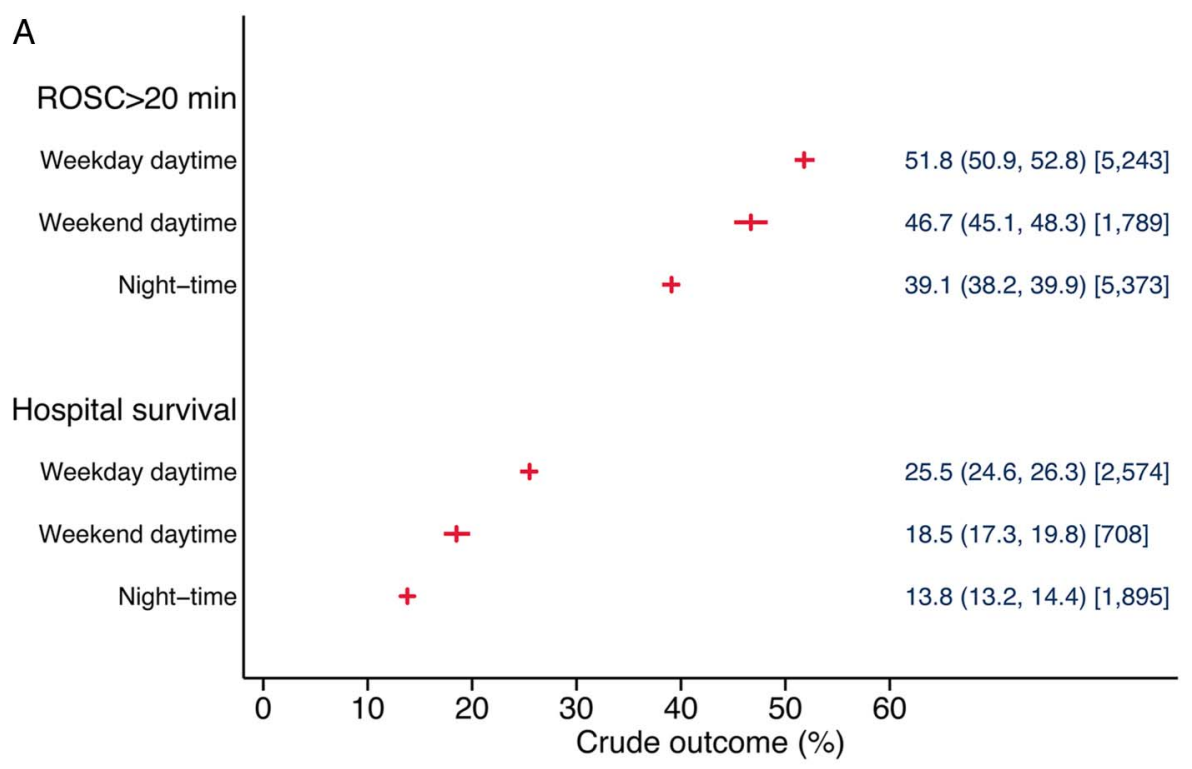

+ Percentage $-95 \% \mathrm{Cl}$

B

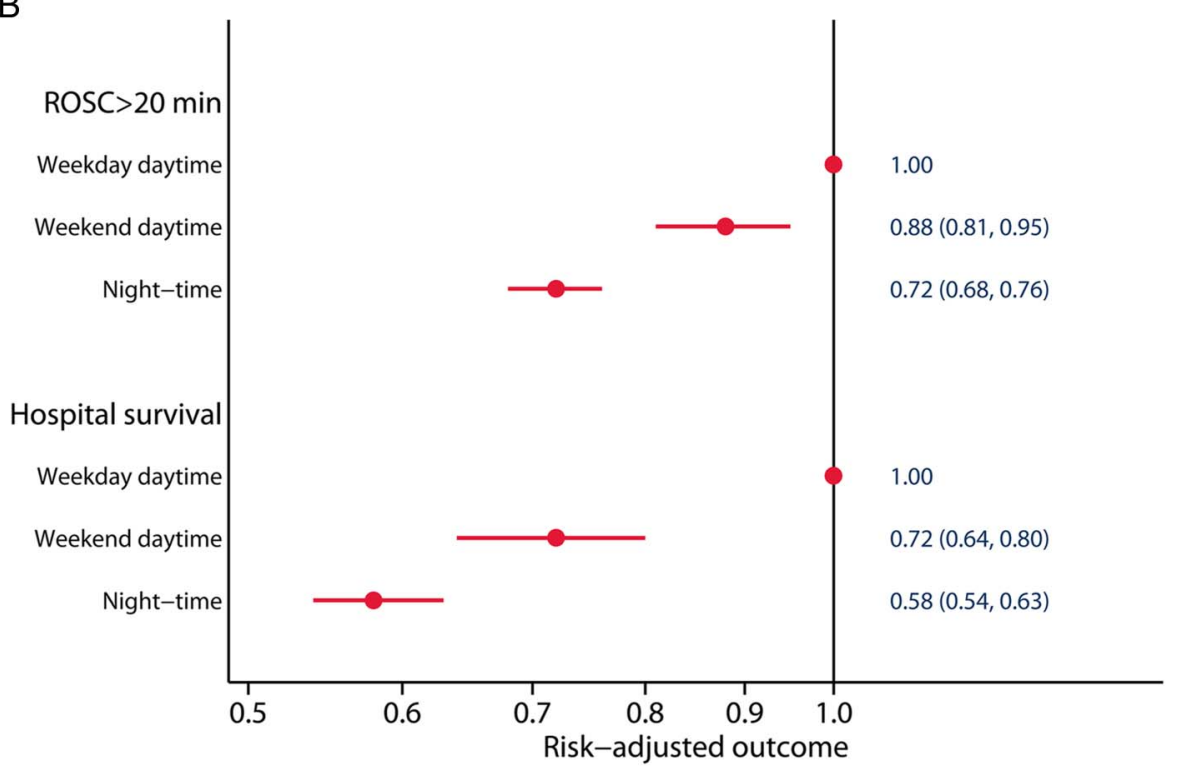

- Odds ratio $-95 \% \mathrm{Cl}$

Figure 3 Crude (A) and risk-adjusted (B) outcomes by day and time of in-hospital cardiac arrest. ROSC, return of spontaneous circulation.

have been admitted on a weekday daytime, but have an IHCA during the weekend daytime or at nights; or be admitted at a weekend and arrest on a weekday daytime. In fact, $>50 \%$ of patients in our study had been in hospital for $\geq 2$ days before having an IHCA. Nevertheless, the question raised by all these studies is whether poorer outcomes are due to differences in patient case mix, administered care or a combination.

We found that IHCAs were equally likely to occur at weekday daytime, weekend daytime and nighttime, and that the measured patient characteristics were broadly similar between the time periods.
Acknowledging that we cannot rule out that differences in unmeasured patient characteristics may contribute, it seems logical to conclude that the differences in outcomes we observed are most likely to be due to care differences. This would seem to echo the work of Concha et $a l,{ }^{4}$ who reported that patients admitted at weekends with cardiac arrest and arrhythmia have an 'early risk' pattern and suggested that their reduced survival is likely to be due to variations in care.

How might differences in care result in different IHCA outcomes? Intuitively, a lack of patient 
A

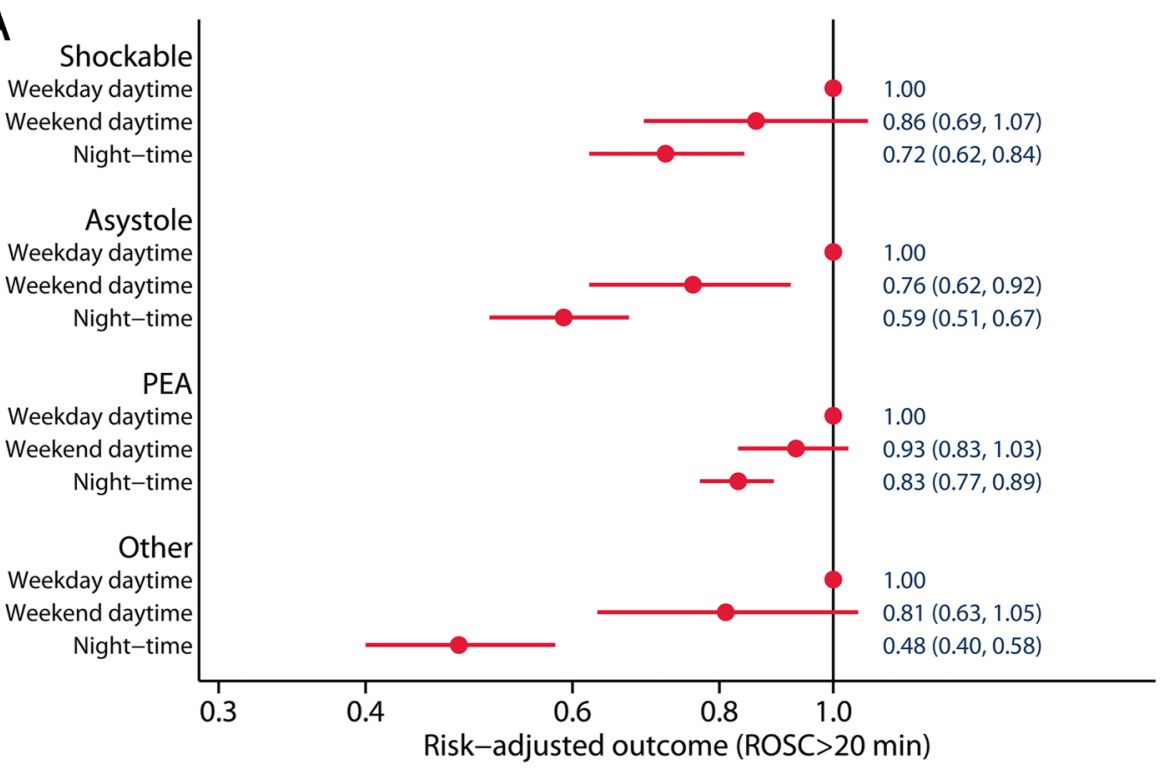

- Odds ratio $-95 \% \mathrm{Cl}$

B

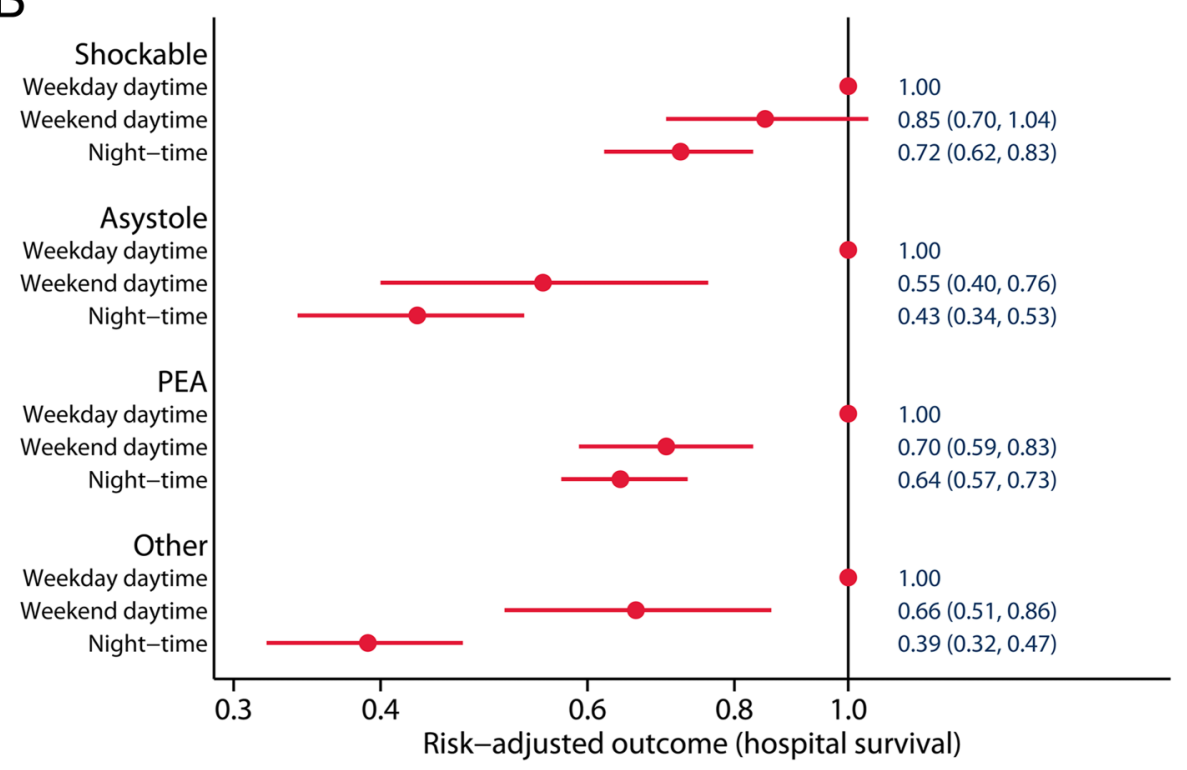

- Odds ratio $=95 \% \mathrm{Cl}$

Figure 4 Risk-adjusted (A) return of spontaneous circulation (ROSC) $>20 \mathrm{~min}$ and (B) acute hospital survival by day and time of in-hospital cardiac arrest and presenting/first documented rhythm. PEA, pulseless electrical activity.

observation or monitoring will reduce opportunities to either prevent an IHCA or detect it early. That non-shockable rhythms are more common at night may reflect delayed detection (it is thought that asystole is a late occurrence in many IHCAs and that some asystolic arrests would be shockable if detected earlier). Data from one study show that vital sign monitoring has a diurnal pattern on all 7 days of the week, with less monitoring at night; ${ }^{25}$ the peak incidence of IHCA in the current and other studies seems also to occur at night. ${ }^{26}$ In our study, most IHCAs occurred on the general ward. In the NHS, the use of continuous monitoring technologies is rare in these areas and most patient monitoring relies upon staff presence.

Fewer nurses at night could also delay the recognition of cardiac arrest, the commencement of life support and the placing of a 2222 call, resulting in poorer outcomes. A recent review reported "...inadequate numbers of nursing staff in a number of ward areas, particularly out of hours-at night and at the weekend..." in some NHS hospitals. ${ }^{27}$ However, the 
UK National Institute for Health and Care Excellence recently reported a lack of high-quality UK studies exploring and quantifying the relationship between nurse staffing levels and skill mix, and outcomes. ${ }^{28}$ Nevertheless, a US study showed that a higher proportion of hours of care provided by registered nurses-a surrogate for the extent of patient observation and care delivery-is associated with lower IHCA rates. ${ }^{29}$ While reduced ward lighting could contribute to delayed detection at night, it would not account for reduced survival at weekend daytime.

The response to a cardiac arrest call might also be slow at night on any day of the week, although evidence about the impact of differing response times through the day is variable. ${ }^{11} 15{ }^{30}$ In the case of medical staff, there is also often a paucity of experienced senior staff immediately available on site, which may influence decision-making before, during, and if successful after, a cardiac arrest.

Any variation in the quality of CPR during the $24 \mathrm{~h}$ period might also explain our findings, although supportive data are sparse. One study suggests that CPR quality is worse at night, ${ }^{31}$ resulting in lower chest compression rates and a significantly larger compression rate variance. Other authors reported that CPR performance and immediate IHCA survival rates at night and during the day were similar. ${ }^{32}$ The quality of postresuscitation care might also vary between day and night; however, the only study exploring this relationship reported no difference in the postresuscitation care provided to survivors of out-of-hospital cardiac arrest. ${ }^{33}$

\section{Future research}

Future research should explore the relationship between staffing (ie, nurse, doctor and allied health practitioner) levels on hospital wards, patient observation and monitoring, and patient outcomes following IHCA. The increasing use of non-invasive, continuous monitoring technologies in the NHS provides opportunities to investigate issues of monitoring further. Future research should also consider the role of other factors that may vary diurnally and that could therefore explain the different survival on weekdays and weekends, and at nights, for example, the composition of the resuscitation team and its access to senior decision makers; the time taken for a functional resuscitation team to assemble and become a cohesive, focused unit; staff tiredness; CPR and other task performance; the situational awareness of staff; and the resuscitation team response times.

\section{CONCLUSIONS}

In conclusion, IHCAs attended by the hospital-based resuscitation team during nights and weekends have substantially worse outcomes than during weekday daytimes. These effects remain significant after risk adjustment and are stronger for non-shockable than shockable rhythms. The precise cause of the observed differences in outcomes cannot be determined by our study as we cannot definitively rule out the effect of unmeasured patient characteristics. However, on the basis of the results obtained, it seems logical to conclude that the worse outcomes at night and weekend daytime are most likely due to organisational or care differences.

Author affiliations

${ }^{1}$ Intensive Care National Audit \& Research Centre, London, UK

${ }^{2}$ Faculty of Health and Social Sciences, University of

Bournemouth, Bournemouth, UK

${ }^{3}$ Department of Anaesthesia and Intensive Care Medicine, Royal United Hospital, Bath, UK

${ }^{4}$ Department of Anaesthesia and Intensive Care Medicine, Southmead Hospital, Bristol, UK

${ }^{5}$ Resuscitation Department, Imperial College Healthcare NHS Trust, London, UK

${ }^{6}$ Resuscitation Council (UK), London, UK

Correction notice This article has been corrected since it was published Online First. The third author's name has been corrected.

Collaborators The National Cardiac Arrest Audit.

Contributors All authors contributed to the study design. Data were collected and cleaned as part of the UK National Cardiac Arrest Audit (NCAA). EJR, GSP and DAH analysed the data and produced the figures and table. All authors contributed to the interpretation of the results and drafting of the manuscript. All authors approved the final version of the manuscript.

Funding This project was supported by internal funding from the Resuscitation Council (UK) and the Intensive Care National Audit \& Research Centre.

Competing interests None declared.

Provenance and peer review Not commissioned; externally peer reviewed.

Open Access This is an Open Access article distributed in accordance with the Creative Commons Attribution Non Commercial (CC BY-NC 4.0) license, which permits others to distribute, remix, adapt, build upon this work noncommercially, and license their derivative works on different terms, provided the original work is properly cited and the use is non-commercial. See: http://creativecommons.org/licenses/by$\mathrm{nc} / 4.0$ /

\section{REFERENCES}

1 Bell CM, Redelmeier DA. Mortality among patients admitted to hospitals on weekends as compared with weekdays. N Engl J Med 2001;345:663-8.

2 Aylin P, Yunus A, Bottle A, et al. Weekend mortality for emergency admissions. A large multicentre study. Qual Saf Health Care 2009;19:213-17.

3 Ruiz M, Bottle A, Aylin PP. The Global Comparators Project: international comparison of 30-day in-hospital mortality by day of the week. BMJ Qual Saf 2015;24:492-504.

4 Concha OP, Gallego B, Hillman K, et al. Do variations in hospital mortality patterns after weekend admission reflect reduced quality of care or different patient cohorts? A population-based study. BMJ Qual Saf 2014;23:215-22.

5 Barba R, Losa JE, Velasco M, et al. Mortality among adult patients admitted to the hospital on weekends. Eur J Intern Med 2006;17:322-4. 
6 Coiera E, Wang Y, Magrabi F, et al. Predicting the cumulative risk of death during hospitalization by modeling weekend, weekday and diurnal mortality risks. BMC Health Serv Res 2014;14:226.

7 Kostis WJ, Demissie K, Marcella SW, et al. Weekend versus weekday admission and mortality from myocardial infarction. N Engl J Med 2007;356:1099-109.

8 Jneid H, Fonarow GC, Cannon CP, et al. Impact of time of presentation on the care and outcomes of acute myocardial infarction. Circulation 2008;117:2502-9.

9 Aylin P, Alexandrescu R, Jen MH, et al. Day of week of procedure and 30 day mortality for elective surgery: retrospective analysis of hospital episode statistics. $B M J$ 2013;346:f2424

10 Goldfrad C, Rowan K. Consequences of discharges from intensive care at night. Lancet 2000;355:1138-42.

11 Herlitz J, Bang A, Alsen B, et al. Characteristics and outcome among patients suffering from in hospital cardiac arrest in relation to whether the arrest took place during office hours. Resuscitation 2002;53:127-33.

12 Matot I, Shleifer A, Hersch M, et al. In-hospital cardiac arrest: is outcome related to the time of arrest? Resuscitation 2006;71:56-64.

13 Brindley PG, Markland DM, Mayers I, et al. Predictors of survival following in-hospital adult cardiopulmonary resuscitation. CMAJ 2002;167:343-8.

14 Jones-Crawford JL, Parish DC, Smith BE, et al. Resuscitation in the hospital: circadian variation of cardiopulmonary arrest. Am J Med 2007;120:158-64.

15 Wright D, Bannister J, Mackintosh AF. Automatic recording and timing of defibrillation on general wards by day and night. Eur Heart J 1994;15:631-6.

16 Dumot JA, Burval DJ, Sprung J, et al. Outcome of adult cardiopulmonary resuscitations at a tertiary referral center including results of "limited" resuscitations. Arch Intern Med 2001;161:1751-8.

17 Nolan JP, Soar J, Smith GB, et al. Incidence and outcome of in-hospital cardiac arrest in the United Kingdom National Cardiac Arrest Audit. Resuscitation 2014;85:987-92.

18 Peberdy MA, Ornato JP, Larkin GL, et al. Survival from in-hospital cardiac arrest during nights and weekends. JAMA 2008;299:785-92.

19 Girotra S, Nallamothu BK, Spertus JA, et al. Trends in survival after in-hospital cardiac arrest. $N$ Engl J Med 2012;367:1912-20.
20 Wunsch H, Angus DC, Harrison DA, et al. Variation in critical care services across North America and Western Europe. Crit Care Med 2008;36:2787-93.

21 Harrison DA, Patel K, Nixon E, et al. Development and validation of risk models to predict outcomes following in-hospital cardiac arrest attended by a hospital-based resuscitation team. Resuscitation 2014;85:993-1000.

22 Chan PS, Berg RA, Spertus JA, et al. for the AHA GWTG-Resuscitation Investigators. Risk-standardizing survival for in-hospital cardiac arrest to facilitate hospital comparisons. J Am Coll Cardiol 2013;62:601-9.

23 Ornstein SM, Nietert PJ, Jenkins RG, et al. The prevalence of chronic diseases and multimorbidity in primary care practice: a PPRNet report. J Am Board Fam Med 2013;26:518-24.

24 Bergeron E, Lavole A, Moore L, et al. Comorbidity and age are both independent predictors of length of hopsitalisation in trauma patients. Can J Surg 2005;48:361-6.

25 Hands C, Reid E, Meredith P, et al. Patterns in the recording of vital signs and early warning scores-time of day, day of week. BMJ Qual Saf 2013;22:719-26.

26 Jones D, Bellomo R, Bates S, et al. Patient monitoring and the timing of cardiac arrests and medical emergency team calls in a teaching hospital. Intensive Care Med 2006;32:1352-6.

27 Keogh B. Review into the quality of care and treatment provided by 14 hospital trusts in England: overview report. NHS England, 2013.

28 National Institute for Health and Clinical Excellence. Safe staffing for nursing in adult inpatient wards in acute hospitals. NICE Safe staffing guideline 1, London, 2014.

29 Needleman J, Buerhaus P, Pankratz VS, et al. Nurse staffing and inpatient hospital mortality. N Engl J Med 2011;364:1037-45.

30 Hajbaghery MA, Mousavi G, Akbari H. Factors influencing survival after in-hospital cardiopulmonary resuscitation. Resuscitation 2005;66:317-21.

31 Perman SM, Smith DA, Leary M, et al. CPR quality is more variable at night than during the day in a multicenter study of inhospital cardiac arrest. Circulation 2010;122:A264.

32 Cooper S, Cade J. Predicting survival, in-hospital cardiac arrests: resuscitation survival variables and training effectiveness. Resuscitation 1997;35:17-22.

33 Uray T, Sterz F, Weiser C, et al. Quality of post arrest care does not differ by time of day at a specialized resuscitation center. Medicine 2015;94:e664. 